\title{
On double Hausdorff summability method
}

\author{
Hamdullah Şevli* and Rabia Savaş
}

"Correspondence:

hsevli@ticaret.edu.tr

Department of Mathematics,

İstanbul Commerce University,

Sütlüce/Beyoğlu, İstanbul, Turkey

\begin{abstract}
Das (Proc. Camb. Philos. Soc. 67:321-326, 1970) proved that every conservative Hausdorff matrix is absolutely kth power conservative. Savaş and Rhoades (Anal. Math. 35:249-256, 2009) proved the result of Das for double Hausdorff summability. In this paper we will consider the double Endl-Jakimovski (E-J) generalization and we will prove the corresponding result of Savaş and Şevli (J. Comput. Anal. Appl. 11:702-710, 2009) for double E-J generalized Hausdorff matrices.
\end{abstract}

MSC: $40 \mathrm{~F} 05 ; 40 \mathrm{G} 05$

Keywords: absolute summability; conservative matrix; double series; Hausdorff matrices

\section{Introduction and background}

The basic theory of Hausdorff transformations for double sequences was developed by Adams [1] in 1933. Later a few authors studied double Hausdorff matrices; see e.g. Ramanujan [2] and Ustina [3].

Several generalizations of Hausdorff matrices have been made. One of them is the EndlJakimovski, or E-J generalization defined independently by Endl [4] and Jakimovski [5] as follows.

Let $\beta$ be a real number, let $\left(\mu_{n}\right)$ be a real sequence, and let $\Delta$ be the forward difference operator defined by $\Delta \mu_{k}=\mu_{k}-\mu_{k+1}, \Delta^{n}\left(\mu_{k}\right)=\Delta\left(\Delta^{n-1} \mu_{k}\right)$. Then the infinite matrix $\left(H^{(\beta)}, \mu_{n}^{(\beta)}\right)=\left(H^{\beta}, \mu\right)=\left(h_{n k}^{(\beta)}\right)$ is defined by

$$
h_{n k}^{(\beta)}= \begin{cases}\left(\begin{array}{l}
n+\beta \\
n-k
\end{array}\right) \Delta^{n-k} \mu_{k}^{(\beta)}, & 0 \leq k \leq n, \\
0, & k>n,\end{cases}
$$

and the associated matrix method is called a generalized Hausdorff matrix and generalized Hausdorff method, respectively. The moment sequence $\mu_{n}^{(\beta)}$ is given by

$$
\mu_{n}^{(\beta)}=\int_{0}^{1} t^{n+\beta} d \chi(t)
$$

where $\chi(t) \in B V[0,1]$. The case $\beta=0$ corresponds to ordinary Hausdorff summability.

In a recent paper [6], the first author jointly with Savaş has extended the result of Das [7] to the E-J matrices; i.e., all conservative E-J matrices are absolutely $k$ th power conservative for $k \geq 1$. Thereafter, Savaş and Rhoades [8] proved the result of Das [7] for double Hausdorff summability. In this paper we will consider double E-J generalization and we will prove the corresponding result of [6] for double E-J generalized Hausdorff matrices. 
Let $\sum_{m=0}^{\infty} \sum_{n=0}^{\infty} a_{m n}$ be an infinite double series with real or complex numbers, with partial sums

$$
s_{m n}=\sum_{i=0}^{m} \sum_{j=0}^{n} a_{i j}
$$

For any double sequence $\left(u_{m n}\right)$ we shall define

$$
\Delta_{11} u_{m n}=u_{m n}-u_{m+1, n}-u_{m, n+1}+u_{m+1, n+1} .
$$

Denote by $\mathcal{A}_{k}^{2}$ the sequence space defined by

$$
\mathcal{A}_{k}^{2}=\left\{\left(s_{m n}\right)_{m, n=0}^{\infty}: \sum_{m=1}^{\infty} \sum_{n=1}^{\infty}(m n)^{k-1}\left|a_{m n}\right|^{k}<\infty ; a_{m n}=\Delta_{11} s_{m-1, n-1}\right\}
$$

for $k \geq 1$

A four-dimensional matrix $T=\left(t_{m n i j}: m, n, i, j=0,1, \ldots\right)$ is said to be absolutely $k$ th power conservative for $k \geq 1$, if $T \in B\left(\mathcal{A}_{k}^{2}\right)$; i.e., if

$$
\sum_{m=1}^{\infty} \sum_{n=1}^{\infty}(m n)^{k-1}\left|\Delta_{11} s_{m-1, n-1}\right|^{k}<\infty
$$

then

$$
\sum_{m=1}^{\infty} \sum_{n=1}^{\infty}(m n)^{k-1}\left|\Delta_{11} t_{m-1, n-1}\right|^{k}<\infty
$$

where

$$
t_{m n}=\sum_{i=0}^{\infty} \sum_{j=0}^{\infty} t_{m n i j} s_{i j} \quad(m, n=0,1, \ldots),
$$

see $e . g .[9,10]$ and the references contained therein.

A double Hausdorff matrix has entries

$$
h_{m n i j}=\left(\begin{array}{c}
m \\
i
\end{array}\right)\left(\begin{array}{c}
n \\
j
\end{array}\right) \Delta_{1}^{m-i} \Delta_{2}^{n-j} \mu_{i j}
$$

where $\left\{\mu_{i j}\right\}$ is any real or complex sequence and

$$
\Delta_{1}^{m-i} \Delta_{2}^{n-j} \mu_{i j}=\sum_{s=0}^{m-i} \sum_{t=0}^{n-j}(-1)^{i+j}\left(\begin{array}{c}
m-i \\
s
\end{array}\right)\left(\begin{array}{c}
n-j \\
t
\end{array}\right) \mu_{i+s \cdot j+t} .
$$

For double Hausdorff matrices, the necessary and sufficient condition for $H$ to be conservative is the existence of a function $\chi(s, t) \in B V[0,1] \times[0,1]$ such that

$$
\int_{0}^{1} \int_{0}^{1}|d \chi(s, t)|<\infty
$$


and

$$
\mu_{m n}=\int_{0}^{1} \int_{0}^{1} s^{m} t^{n} d \chi(s, t)
$$

Quite recently, Savaş and Rhoades [8] extended the result of Das [7] to double Hausdorff summability. Their theorem is as follows.

Theorem 1 [8] Let $H$ be a conservative double Hausdorff matrix. Then $H \in B\left(\mathcal{A}_{k}^{2}\right)$.

Our purpose is to achieve the result established in [7] for double E-J Hausdorff summability.

\section{Main results}

The matrix $\delta^{(\alpha, \beta)}=\left(\delta_{m n i j}^{(\alpha, \beta)}\right)$, whose elements are defined by

$$
\delta_{m n i j}^{(\alpha, \beta)}= \begin{cases}(-1)^{i+j}\left(\begin{array}{c}
m+\alpha \\
m-i
\end{array}\right)\left(\begin{array}{c}
n+\beta \\
n-j
\end{array}\right), & i \leq m, j \leq n, \\
0, & \text { otherwise }\end{cases}
$$

is called a difference matrix, where $\alpha$ and $\beta$ are real numbers.

Theorem 2 The difference matrix $\delta^{(\alpha, \beta)}=\left(\delta_{m n i j}^{(\alpha, \beta)}\right)$ is its own inverse.

Proof Let

$$
a_{m n k l}=\sum_{i=0}^{m} \sum_{j=0}^{n} \delta_{m n i j}^{(\alpha, \beta)} \delta_{i j k l}^{(\alpha, \beta)}
$$

thus $A=\delta^{(\alpha, \beta)} \delta^{(\alpha, \beta)}$. For any double sequence $\left(u_{r s}\right)$

$$
\begin{aligned}
& \sum_{r=0}^{m} \sum_{s=0}^{n} a_{m n r s} u_{r s} \\
& \quad=\sum_{r=0}^{m} \sum_{s=0}^{n} \sum_{i=0}^{m} \sum_{j=0}^{n} \delta_{m n i j}^{(\alpha, \beta)} \delta_{i j r s}^{(\alpha, \beta)} u_{r s} \\
& \quad=\sum_{r=0}^{m} \sum_{s=0}^{n}(-1)^{r+s} u_{r s} \sum_{i=r}^{m} \sum_{j=s}^{n}(-1)^{i+j}\left(\begin{array}{c}
m+\alpha \\
m-i
\end{array}\right)\left(\begin{array}{c}
n+\beta \\
n-j
\end{array}\right)\left(\begin{array}{c}
i+\alpha \\
i-r
\end{array}\right)\left(\begin{array}{c}
j+\beta \\
j-s
\end{array}\right) \\
& \quad=\sum_{r=0}^{m} \sum_{s=0}^{n}(-1)^{r+s} u_{r s}\left(\begin{array}{c}
m+\alpha \\
m-r
\end{array}\right)\left(\begin{array}{c}
n+\beta \\
n-s
\end{array}\right) \sum_{i=r}^{m} \sum_{j=s}^{n}(-1)^{i+j}\left(\begin{array}{c}
m-r \\
m-i
\end{array}\right)\left(\begin{array}{c}
n-s \\
n-j
\end{array}\right) \\
& \quad=u_{r s},
\end{aligned}
$$

since

$$
\sum_{i=r}^{m} \sum_{j=s}^{n}(-1)^{i+j}\left(\begin{array}{l}
m-r \\
m-i
\end{array}\right)\left(\begin{array}{l}
n-s \\
n-j
\end{array}\right)= \begin{cases}(-1)^{r+s}, & m=r, n=s \\
0, & \text { otherwise. }\end{cases}
$$


Let $\left(\mu_{m n}^{(\alpha, \beta)}\right)$ be a given sequence and $\mu^{(\alpha, \beta)}=\left(\mu_{m n i j}^{(\alpha, \beta)}\right)$ be a diagonal matrix whose only non-zero entries are $\mu_{m n}^{(\alpha, \beta)}=\mu_{m n m n}^{(\alpha, \beta)}$. The transformation matrix

$$
H^{(\alpha, \beta)}=\delta^{(\alpha, \beta)} \mu^{(\alpha, \beta)} \delta^{(\alpha, \beta)}
$$

is called a double E-J generalized Hausdorff matrix corresponding to the sequence $\left(\mu_{m n}^{(\alpha, \beta)}\right)$.

Theorem 3 A matrix $H^{(\alpha, \beta)}=\left(h_{m n i j}^{(\alpha, \beta)}\right)$ is a double E-J generalized Hausdorff matrix corresponding to the sequence $\left(\mu_{m n}^{(\alpha, \beta)}\right)$ if and only if its elements have the form

$$
h_{m n i j}^{(\alpha, \beta)}=\left(\begin{array}{c}
m+\alpha \\
m-i
\end{array}\right)\left(\begin{array}{c}
n+\beta \\
n-j
\end{array}\right) \Delta_{1}^{m-i} \Delta_{2}^{n-j} \mu_{i j}^{(\alpha, \beta)}
$$

where

$$
\Delta_{1}^{m-i} \Delta_{2}^{n-j} \mu_{i j}^{(\alpha, \beta)}:=\sum_{r=0}^{m-i} \sum_{s=0}^{n-j}(-1)^{r+s}\left(\begin{array}{c}
m-i \\
r
\end{array}\right)\left(\begin{array}{c}
n-j \\
s
\end{array}\right) \mu_{i+r, j+s}^{(\alpha, \beta)}
$$

Proof Let $H^{(\alpha, \beta)}=\delta^{(\alpha, \beta)} \mu^{(\alpha, \beta)} \delta^{(\alpha, \beta)}$ be a double E-J Hausdorff matrix. Applying this to a double sequence $\left(s_{m n}\right)$ we have

$$
\begin{aligned}
t_{m n} & =\sum_{i=0}^{m} \sum_{j=0}^{n} h_{m n i j}^{(\alpha, \beta)} s_{i j} \\
& =\sum_{i=0}^{m} \sum_{j=0}^{n} \sum_{r=0}^{m} \sum_{s=0}^{n} \delta_{m n r s}^{(\alpha, \beta)} \mu_{r s}^{(\alpha, \beta)} \delta_{r s i j}^{(\alpha, \beta)} s_{i j} \\
& =\sum_{i=0}^{m} \sum_{j=0}^{n} \sum_{r=0}^{m} \sum_{s=0}^{n}(-1)^{r+s}\left(\begin{array}{c}
m+\alpha \\
m-r
\end{array}\right)\left(\begin{array}{c}
n+\beta \\
n-s
\end{array}\right) \mu_{r s}^{(\alpha, \beta)}(-1)^{i+j}\left(\begin{array}{c}
r+\alpha \\
r-i
\end{array}\right)\left(\begin{array}{c}
s+\beta \\
s-j
\end{array}\right) s_{i j} \\
& =\sum_{i=0}^{m} \sum_{j=0}^{n}(-1)^{i+j}\left(\begin{array}{c}
m+\alpha \\
m-i
\end{array}\right)\left(\begin{array}{c}
n+\beta \\
n-j
\end{array}\right) \sum_{r=i}^{m} \sum_{s=j}^{n}(-1)^{r+s}\left(\begin{array}{c}
m-i \\
m-r
\end{array}\right)\left(\begin{array}{c}
n-j \\
n-s
\end{array}\right) \mu_{r s}^{(\alpha, \beta)} s_{i j} \\
& =\sum_{i=0}^{m} \sum_{j=0}^{n}\left(\begin{array}{c}
m+\alpha \\
m-i
\end{array}\right)\left(\begin{array}{c}
n+\beta \\
n-j
\end{array}\right) \sum_{r=0}^{m-i} \sum_{s=0}^{n-j}(-1)^{r+s}\left(\begin{array}{c}
m-i \\
r
\end{array}\right)\left(\begin{array}{c}
n-j \\
s
\end{array}\right) \mu_{i+r, j+s}^{(\alpha, \beta)} s_{i j} .
\end{aligned}
$$

Hence

$$
h_{m n i j}^{(\alpha, \beta)}=\left(\begin{array}{c}
m+\alpha \\
m-i
\end{array}\right)\left(\begin{array}{c}
n+\beta \\
n-j
\end{array}\right) \sum_{r=0}^{m-i} \sum_{s=0}^{n-j}(-1)^{r+s}\left(\begin{array}{c}
m-i \\
r
\end{array}\right)\left(\begin{array}{c}
n-j \\
s
\end{array}\right) \mu_{i+r, j+s}^{(\alpha, \beta)} .
$$

For double E-J Hausdorff matrices, the necessary and sufficient condition for $H^{(\alpha, \beta)}$ to be conservative is the existence of a function $\chi(s, t) \in B V[0,1] \times[0,1]$ such that

$$
\int_{0}^{1} \int_{0}^{1}|d \chi(s, t)|<\infty
$$

and

$$
\mu_{m n}^{(\alpha, \beta)}=\int_{0}^{1} \int_{0}^{1} s^{m+\alpha} t^{n+\beta} d \chi(s, t)
$$


Theorem 4 Given a function $\chi(s, t) \in B V[0,1] \times[0,1]$, a bounded variation in the unit square, the corresponding double E-J Hausdorff transformation $\left(t_{m n}\right)$, of a sequence $\left(s_{m n}\right)$, may be defined by

$$
t_{m n}=\sum_{i=0}^{m} \sum_{j=0}^{n}\left(\begin{array}{c}
m+\alpha \\
m-i
\end{array}\right)\left(\begin{array}{c}
n+\beta \\
n-j
\end{array}\right) s_{i j} \int_{0}^{1} \int_{0}^{1} s^{i+\alpha}(1-s)^{m-i} t^{j+\beta}(1-t)^{n-j} d \chi(s, t) .
$$

Proof For $i \leq m$ and $j \leq n$,

$$
\begin{aligned}
h_{m n i j}^{(\alpha, \beta)} & =\sum_{k=i}^{m} \sum_{l=j}^{n} \delta_{m n k l}^{(\alpha, \beta)} \mu_{k l}^{(\alpha, \beta)} \delta_{k l i j}^{(\alpha, \beta)} \\
& =\sum_{k=i}^{m} \sum_{l=j}^{n} \delta_{m n k l}^{(\alpha, \beta)} \int_{0}^{1} \int_{0}^{1} s^{k+\alpha} t^{l+\beta} d \chi(s, t) \cdot \delta_{k l i j}^{(\alpha, \beta)} \\
& =\int_{0}^{1} \int_{0}^{1} \sum_{k=i}^{m} \sum_{l=j}^{n}(-1)^{k+l}\left(\begin{array}{c}
m+\alpha \\
m-k
\end{array}\right)\left(\begin{array}{c}
n+\beta \\
n-l
\end{array}\right)(-1)^{i+j}\left(\begin{array}{c}
k+\alpha \\
k-i
\end{array}\right)\left(\begin{array}{c}
l+\beta \\
l-j
\end{array}\right) s^{k+\alpha} t^{l+\beta} d \chi(s, t) \\
& =\int_{0}^{1} \int_{0}^{1} \sum_{k=i}^{m} \sum_{l=j}^{n}(-1)^{k+l+i+j}\left(\begin{array}{c}
m+\alpha \\
m-i
\end{array}\right)\left(\begin{array}{c}
n+\beta \\
n-j
\end{array}\right)\left(\begin{array}{c}
m-i \\
m-k
\end{array}\right)\left(\begin{array}{c}
n-j \\
n-l
\end{array}\right) s^{k+\alpha} t^{l+\beta} d \chi(s, t) \\
& =\int_{0}^{1} \int_{0}^{1}\left(\begin{array}{c}
m+\alpha \\
m-i
\end{array}\right)\left(\begin{array}{c}
n+\beta \\
n-j
\end{array}\right) \sum_{k=0}^{m-i} \sum_{l=0}^{n-j}(-1)^{k+l}\left(\begin{array}{c}
m-i \\
k
\end{array}\right)\left(\begin{array}{c}
n-j \\
l
\end{array}\right) s^{k+i+\alpha} t^{l+j+\beta} d \chi(s, t) \\
& =\int_{0}^{1} \int_{0}^{1}\left(\begin{array}{c}
m+\alpha \\
m-i
\end{array}\right)\left(\begin{array}{c}
n+\beta \\
n-j
\end{array}\right) s^{i+\alpha} t^{j+\beta}\left(\sum_{k=0}^{m-i} \sum_{l=0}^{n-j}(-1)^{k+l}\left(\begin{array}{c}
m-i \\
k
\end{array}\right)\left(\begin{array}{c}
n-j \\
l
\end{array}\right) s^{k} t^{l}\right) d \chi(s, t) \\
& =\int_{0}^{1} \int_{0}^{1}\left(\begin{array}{c}
m+\alpha \\
m-i
\end{array}\right)\left(\begin{array}{c}
n+\beta \\
n-j
\end{array}\right) s^{i+\alpha} t^{j+\beta}(1-s)^{m-i}(1-t)^{n-j} d \chi(s, t) .
\end{aligned}
$$

Theorem 5 Let $H^{(\alpha, \beta)}$ be a conservative double E-JHausdorff matrix. Then $H^{(\alpha, \beta)} \in B\left(\mathcal{A}_{k}^{2}\right)$, $\alpha, \beta \geq 0$.

As tools to prove our result, we need to the following lemmas.

Lemma 1 [6] Let $k \geq 1, n \geq v$ and $\alpha \geq 0$. Then

$$
E_{m+\alpha}^{k-1} E_{m-\mu}^{\mu+\alpha-1} \leq E_{\mu+\alpha}^{k-1} E_{m-\mu}^{\mu+\alpha+k-2}
$$

The following lemma is a double version of [11].

Lemma 2 For $0 \leq s \leq 1,0 \leq t \leq 1, \alpha \geq 0$ and $\beta \geq 0$

$$
\sum_{i=0}^{m} \sum_{j=0}^{n}\left(\begin{array}{c}
m+\alpha \\
i
\end{array}\right)\left(\begin{array}{c}
n+\beta \\
j
\end{array}\right)(1-s)^{m}(1-t)^{n} s^{m+\alpha-i} t^{n+\beta-j} \leq 1 .
$$

Proof of Theorem 5 Let $\left(t_{m n}\right)$ be the double E-J transform of a double sequence $\left(s_{m n}\right)$; i.e.,

$$
t_{m n}=\sum_{\mu=0}^{m} \sum_{v=0}^{n} h_{m n \mu \nu}^{(\alpha, \beta)} s_{\mu \nu}
$$


We will demonstrate that

$$
\sum_{m=1}^{\infty} \sum_{n=1}^{\infty}(m n)^{k-1}\left|a_{m n}\right|^{k}<\infty \Rightarrow \sum_{m=1}^{\infty} \sum_{n=1}^{\infty}(m n)^{k-1}\left|\Delta_{11} t_{m-1, n-1}\right|^{k}<\infty .
$$

Write

$$
t_{m n}=\sum_{\mu=0}^{m} \sum_{v=0}^{n} b_{\mu \nu} .
$$

Then $b_{m n}=\Delta_{11} t_{m-1, n-1}$. For $k \geq 1$

$$
E_{m}^{k-1}=\left(\begin{array}{c}
m+k-1 \\
m
\end{array}\right)=\left(\begin{array}{c}
m+k-1 \\
k-1
\end{array}\right)=\frac{(m+k-1) !}{m !(k-1) !}=\frac{\Gamma(m+k)}{\Gamma(m+1) \Gamma(k)} .
$$

Then

$$
\begin{aligned}
& E_{m}^{k-1} \approx \frac{m^{k-1}}{\Gamma(k)} \approx m^{k-1}, \\
& m^{k-1} \approx E_{m}^{k-1} \approx E_{m+\alpha}^{k-1} .
\end{aligned}
$$

Due to this (1) is equivalent to

$$
\sum_{m=1}^{\infty} \sum_{n=1}^{\infty} E_{m+\alpha}^{k-1} E_{n+\beta}^{k-1}\left|a_{m n}\right|^{k}<\infty \Rightarrow \sum_{m=1}^{\infty} \sum_{n=1}^{\infty} E_{m+\alpha}^{k-1} E_{n+\beta}^{k-1}\left|b_{m n}\right|^{k}<\infty .
$$

For $s \in[0,1]$ and $t \in[0,1]$ define

$$
\phi_{m n}(s, t)=\sum_{\mu=1}^{m} \sum_{v=1}^{n} E_{m-\mu}^{\mu+\alpha-1} E_{n-v}^{v+\beta-1} s^{\mu+\alpha} t^{\nu+\beta}(1-s)^{m-\mu}(1-t)^{n-v} a_{\mu \nu} .
$$

It follows from the Hölder inequality that

$$
\begin{aligned}
\left|\phi_{m n}(s, t)\right|^{k}= & \left|\sum_{\mu=1}^{m} \sum_{v=1}^{n} E_{m-\mu}^{\mu+\alpha-1} E_{n-\nu}^{\nu+\beta-1} s^{\mu+\alpha} t^{\nu+\beta}(1-s)^{m-\mu}(1-t)^{n-v} a_{\mu \nu}\right|^{k} \\
\leq & \sum_{\mu=1}^{m} \sum_{\nu=1}^{n} E_{m-\mu}^{\mu+\alpha-1} E_{n-\nu}^{\nu+\beta-1} s^{\mu+\alpha} t^{\nu+\beta}(1-s)^{m-\mu}(1-t)^{n-v}\left|a_{\mu \nu}\right|^{k} \\
& \times\left\{\sum_{\mu=1}^{m} \sum_{v=1}^{n} E_{m-\mu}^{\mu+\alpha-1} E_{n-\nu}^{\nu+\beta-1} s^{\mu+\alpha} t^{\nu+\beta}(1-s)^{m-\mu}(1-t)^{n-\nu}\right\}^{k-1} .
\end{aligned}
$$

From Lemma 2

$$
\begin{aligned}
\sum_{\mu=1}^{m} & \sum_{v=1}^{n} E_{m-\mu}^{\mu+\alpha-1} E_{n-v}^{v+\beta-1} s^{\mu+\alpha} t^{\nu+\beta}(1-s)^{m-\mu}(1-t)^{n-v} \\
\quad & \sum_{\mu=1}^{m} \sum_{v=1}^{n}\left(\begin{array}{c}
m+\alpha-1 \\
m-\mu
\end{array}\right)\left(\begin{array}{c}
n+\beta-1 \\
n-v
\end{array}\right) s^{\mu+\alpha} t^{\nu+\beta}(1-s)^{m-\mu}(1-t)^{n-v}
\end{aligned}
$$




$$
\begin{aligned}
& =\sum_{\mu=0}^{m-1} \sum_{\nu=0}^{n-1}\left(\begin{array}{c}
m+\alpha-1 \\
m-\mu-1
\end{array}\right)\left(\begin{array}{c}
n+\beta-1 \\
n-v-1
\end{array}\right) s^{\mu+\alpha+1} t^{\nu+\beta+1}(1-s)^{m-\mu-1}(1-t)^{n-\nu-1} \\
& =s t \sum_{\mu=0}^{m-1} \sum_{\nu=0}^{n-1}\left(\begin{array}{c}
m+\alpha-1 \\
m-\mu-1
\end{array}\right)\left(\begin{array}{c}
n+\beta-1 \\
n-v-1
\end{array}\right) s^{\mu+\alpha} t^{\nu+\beta}(1-s)^{m-\mu-1}(1-t)^{n-\nu-1} \\
& =O(s t) .
\end{aligned}
$$

Hence

$$
\left|\phi_{m n}(s, t)\right|^{k}=O(1)(s t)^{k-1} \sum_{\mu=1}^{m} \sum_{\nu=1}^{n} E_{m-\mu}^{\mu+\alpha-1} E_{n-v}^{\nu+\beta-1} s^{\mu+\alpha} t^{\nu+\beta}(1-s)^{m-\mu}(1-t)^{n-v}\left|a_{\mu \nu}\right|^{k}
$$

and from Lemma 1

$$
\begin{aligned}
& \sum_{m=1}^{\infty} \sum_{n=1}^{\infty} E_{m+\alpha}^{k-1} E_{n+\beta}^{k-1}\left|\phi_{m n}(s, t)\right|^{k} \\
& =O(1) \sum_{m=1}^{\infty} \sum_{n=1}^{\infty} E_{m+\alpha}^{k-1} E_{n+\beta}^{k-1}(s t)^{k-1} \\
& \times \sum_{\mu=1}^{m} \sum_{\nu=1}^{n} E_{m-\mu}^{\mu+\alpha-1} E_{n-v}^{\nu+\beta-1} s^{\mu+\alpha} t^{\nu+\beta}(1-s)^{m-\mu}(1-t)^{n-v}\left|a_{\mu \nu}\right|^{k} \\
& =O(1)(s t)^{k-1} \sum_{\mu=1}^{\infty} \sum_{\nu=1}^{\infty} s^{\mu+\alpha} t^{\nu+\beta}\left|a_{\mu \nu}\right|^{k} \\
& \times \sum_{m=\mu}^{\infty} \sum_{n=v}^{\infty} E_{m+\alpha}^{k-1} E_{n+\beta}^{k-1} E_{m-\mu}^{\mu+\alpha-1} E_{n-v}^{\nu+\beta-1}(1-s)^{m-\mu}(1-t)^{n-v} \\
& =O(1)(s t)^{k-1} \sum_{\mu=1}^{\infty} \sum_{\nu=1}^{\infty} s^{\mu+\alpha} t^{\nu+\beta}\left|a_{\mu \nu}\right|^{k} E_{\mu+\alpha}^{k-1} E_{v+\beta}^{k-1} \\
& \times \sum_{m=\mu}^{\infty} \sum_{n=v}^{\infty} E_{m-\mu}^{\mu+\alpha+k-2} E_{n-\nu}^{\nu+\beta+k-2}(1-s)^{m-\mu}(1-t)^{n-v} \\
& =O(1)(s t)^{k-1} \sum_{\mu=1}^{\infty} \sum_{\nu=1}^{\infty} s^{\mu+\alpha} t^{\nu+\beta}\left|a_{\mu \nu}\right|^{k} E_{\mu+\alpha}^{k-1} E_{\nu+\beta}^{k-1} s^{-\mu-\alpha-k+1} t^{-v-\beta-k+1} \\
& =O(1) \sum_{\mu=1}^{\infty} \sum_{v=1}^{\infty} E_{\mu+\alpha}^{k-1} E_{v+\beta}^{k-1}\left|a_{\mu \nu}\right|^{k} \text {. }
\end{aligned}
$$

From Lemma of [8], if $\left(t_{m n}\right)$ and $\left(\tau_{m n}\right)$ are the $\left(H, \mu_{m n}\right)$ transformation of $\left(s_{m n}\right)$ and $\left(m n a_{m n}\right)$, respectively, then

$$
\tau_{m n}=m n \Delta_{11} t_{m-1, n-1} .
$$

A similar consequence can be proved for $\left(H^{(\alpha, \beta)}, \mu^{(\alpha, \beta)}\right)$, see [6]; i.e.,

$$
\tau_{m n}=(m+\alpha)(n+\beta) \Delta_{11} t_{m-1, n-1} .
$$


Hence

$$
\begin{aligned}
b_{m n} & =\frac{1}{(m+\alpha)(n+\beta)} \tau_{m n} \\
& =\frac{1}{(m+\alpha)(n+\beta)} \sum_{i=0}^{m} \sum_{j=0}^{n}\left(\begin{array}{c}
m+\alpha \\
m-i
\end{array}\right)\left(\begin{array}{c}
n+\beta \\
n-j
\end{array}\right) \Delta_{1}^{m-i} \Delta_{2}^{n-j} \mu_{i j}^{(\alpha, \beta)}(i+\alpha)(j+\beta) a_{i j} \\
& =\sum_{i=0}^{m} \sum_{j=0}^{n}\left(\begin{array}{c}
m+\alpha-1 \\
m-i
\end{array}\right)\left(\begin{array}{c}
n+\beta-1 \\
n-j
\end{array}\right) \Delta_{1}^{m-i} \Delta_{2}^{n-j} \mu_{i j}^{(\alpha, \beta)} a_{i j} \\
& =\sum_{i=0}^{m} \sum_{j=0}^{n} E_{m-i}^{i+\alpha-1} E_{n-j}^{j+\beta-1} \Delta_{1}^{m-i} \Delta_{2}^{n-j} \mu_{i j}^{(\alpha, \beta)} a_{i j} .
\end{aligned}
$$

Since $H^{(\alpha, \beta)}$ is conservative, $\mu_{n}^{(\alpha, \beta)}$ is a moment sequence,

$$
\mu_{m n}^{(\alpha, \beta)}=\int_{0}^{1} \int_{0}^{1} s^{m+\alpha} t^{n+\beta} d \chi(s, t)
$$

and

$$
\Delta_{1}^{m-i} \Delta_{2}^{n-j} \mu_{i j}^{(\alpha, \beta)}=\int_{0}^{1} \int_{0}^{1} s^{i+\alpha}(1-s)^{m-i} t^{j+\beta}(1-t)^{n-j} d \chi(s, t)
$$

from Theorem 4 . In view of (3) we can deduce that

$$
\begin{aligned}
b_{m n} & =\sum_{i=0}^{m} \sum_{j=0}^{n} E_{m-i}^{i+\alpha-1} E_{n-j}^{j+\beta-1} \int_{0}^{1} \int_{0}^{1} s^{i+\alpha}(1-s)^{m-i} t^{j+\beta}(1-t)^{n-j} d \chi(s, t) a_{i j} \\
& =\int_{0}^{1} \int_{0}^{1}\left(\sum_{i=0}^{m} \sum_{j=0}^{n} E_{m-i}^{i+\alpha-1} E_{n-j}^{j+\beta-1} s^{i+\alpha} t^{j+\beta}(1-s)^{m-i}(1-t)^{n-j} a_{i j}\right) d \chi(s, t) \\
& =\int_{0}^{1} \int_{0}^{1} \phi_{m n}(s, t) d \chi(s, t) .
\end{aligned}
$$

Using Minkowski's inequality we get

$$
\begin{aligned}
\left\{\sum_{m=1}^{\infty} \sum_{n=1}^{\infty} E_{m+\alpha}^{k-1} E_{n+\beta}^{k-1}\left|b_{m n}\right|^{k}\right\}^{1 / k} & =\left\{\sum_{m=1}^{\infty} \sum_{n=1}^{\infty} E_{m+\alpha}^{k-1} E_{n+\beta}^{k-1}\left|\int_{0}^{1} \int_{0}^{1} \phi_{m n}(s, t) d \chi(s, t)\right|^{k}\right\}^{1 / k} \\
& \leq \int_{0}^{1} \int_{0}^{1}|d \chi(s, t)|\left\{\sum_{m=1}^{\infty} \sum_{n=1}^{\infty} E_{m+\alpha}^{k-1} E_{n+\beta}^{k-1}\left|\phi_{m n}(s, t)\right|^{k}\right\}^{1 / k} \\
& =O(1) \int_{0}^{1} \int_{0}^{1}|d \chi(s, t)|\left\{\sum_{\mu=1}^{\infty} \sum_{\nu=1}^{\infty} E_{\mu+\alpha}^{k-1} E_{v+\beta}^{k-1}\left|a_{\mu \nu}\right|^{k}\right\}^{1 / k} .
\end{aligned}
$$

Therefore the proof of Theorem 5 is complete.

Specially, if we take $\alpha=0$ and $\beta=0$ in Theorem 5 , we get Theorem 1 as a corollary. The following is an example of a double E-J Hausdorff matrix. 
A doubly infinite Cesàro matrix $(C, \gamma, \delta)$ is a doubly infinite Hausdorff matrix with entries

$$
h_{m n i j}=\frac{\left(\begin{array}{c}
m+\gamma-i-1 \\
n-i
\end{array}\right)\left(\begin{array}{c}
n+\delta-j-1 \\
n-j
\end{array}\right)}{\left(\begin{array}{c}
m+\gamma \\
\gamma
\end{array}\right)\left(\begin{array}{c}
n+\delta \\
\delta
\end{array}\right)}, \quad \gamma, \delta \geq 0 .
$$

We use the following to denote the corresponding E-J generalizations of the $(C, \gamma, \delta)$. $\left(C^{(\alpha, \beta)}, \gamma, \delta\right)$ has moment sequence

$$
\mu_{m n}^{(\alpha, \beta)}=\int_{0}^{1} \int_{0}^{1} u^{m+\alpha} v^{n+\beta} \gamma \delta(1-u)^{\gamma-1}(1-v)^{\delta-1} d u d v
$$

where

$$
\chi(u, v)=\gamma \delta \int_{0}^{u} \int_{0}^{v}(1-s)^{\gamma-1}(1-t)^{\delta-1} d s d t .
$$

For $i \leq m$ and $j \leq n$,

$$
\begin{aligned}
h_{m n i j}^{(\alpha, \beta)}= & \int_{0}^{1} \int_{0}^{1}\left(\begin{array}{c}
m+\alpha \\
m-i
\end{array}\right)\left(\begin{array}{c}
n+\beta \\
n-j
\end{array}\right) u^{i+\alpha} v^{j+\beta}(1-u)^{m-i}(1-v)^{n-j} d \chi(u, v) \\
= & \int_{0}^{1} \int_{0}^{1}\left(\begin{array}{c}
m+\alpha \\
m-i
\end{array}\right)\left(\begin{array}{c}
n+\beta \\
n-j
\end{array}\right) \\
& \times u^{i+\alpha} v^{j+\beta}(1-u)^{m-i}(1-v)^{n-j} \gamma \delta(1-u)^{\gamma-1}(1-v)^{\delta-1} d u d v \\
= & \gamma \delta\left(\begin{array}{c}
m+\alpha \\
m-i
\end{array}\right)\left(\begin{array}{c}
n+\beta \\
n-j
\end{array}\right) \int_{0}^{1} \int_{0}^{1} u^{i+\alpha}(1-u)^{m-i+\gamma-1} v^{j+\beta}(1-v)^{n-j+\delta-1} d u d v \\
= & \gamma \delta\left(\begin{array}{c}
m+\alpha \\
m-i
\end{array}\right)\left(\begin{array}{c}
n+\beta \\
n-j
\end{array}\right) B(i+\alpha+1, m-i+\gamma) B(j+\beta+1, n-j+\delta) \\
= & \frac{\gamma \Gamma(m+\alpha+1) \Gamma(m-i+\gamma)}{\Gamma(m-i+1) \Gamma(m+\alpha+\gamma+1)} \frac{\delta \Gamma(n+\beta+1) \Gamma(n-j+\delta)}{\Gamma(n-j+1) \Gamma(n+\beta+\delta+1)} \\
= & \frac{E_{m-i}^{\gamma-1} E_{n-j}^{\delta-1}}{E_{m+\alpha}^{\gamma} E_{n+\beta}^{\delta}}
\end{aligned}
$$

For the special case $\gamma, \delta=1$,

$$
\left(C^{(\alpha, \beta)}, 1,1\right)= \begin{cases}\frac{1}{(m+\alpha+1)(n+\beta+1)}, & i \leq m \text { and } j \leq n, \\ 0, & \text { otherwise }\end{cases}
$$

is a double E-J Hausdorff matrix.

\section{Competing interests}

The authors declare that they have no competing interests.

\section{Authors' contributions}

The authors contributed equally and significantly in writing this paper. Both authors read and approved the final manuscript.

\section{Acknowledgements}

This work is supported by Istanbul Commerce University Scientific Research Projects Coordination Unit. 


\section{References}

1. Adams, CR: Hausdorff transformations for double sequences. Bull. Am. Math. Soc. 39, 303-312 (1933)

2. Ramanujan, MS: On Hausdorff transformations for double sequences. Proc. Indian Acad. Sci. Sect. A. 42, 131-135 (1955)

3. Ustina, F: The Hausdorff means for double sequences. Can. Math. Bull. 10, 347-352 (1967)

4. Endl, K: Untersuchungen über momentenprobleme bei verfahren vom Hausdorffschen typus. Math. Ann. 139, 403-432 (1960)

5. Jakimovski, A: The product of summability methods; part 2. Technical Report 8, Jerusalem (1959)

6. Savaş, E, Şevli, H: Generalized Hausdorff matrices as bounded operators over $\mathcal{A}_{k}$. J. Comput. Anal. Appl. 11, 702-710 (2009)

7. Das, G: A Tauberian theorem for absolute summability. Proc. Camb. Philos. Soc. 67, 321-326 (1970)

8. Savaş, E, Rhoades, BE: Every conservative double Hausdorff matrix is a $k$-th absolutely summable operator. Anal. Math. 35, 249-256 (2009)

9. Savaş, E, Şevli, H: On absolute summability for double triangle matrices. Math. Slovaca 60, 495-506 (2010)

10. Sevli, H, Savaș, E: Some further extensions of absolute Cesàro summability for double series. J. Inequal. Appl. 2013, 144 (2013)

11. Jakimovski, A, Ramanujan, MS: A uniform approximation theorem and its application to moment problems. Math. Z 84, 143-153 (1964)

doi:10.1186/1029-242X-2014-240

Cite this article as: Sevli and Savaș: On double Hausdorff summability method. Journal of Inequalities and Applications 2014 2014:240.

\section{Submit your manuscript to a SpringerOpen ${ }^{\ominus}$ journal and benefit from:}

- Convenient online submission

- Rigorous peer review

- Immediate publication on acceptance

- Open access: articles freely available online

- High visibility within the field

- Retaining the copyright to your article 